\title{
Gestión de la sostenibilidad portuaria basada en un modelo de redes bayesianas. Aplicación al sistema portuario español
}

\author{
Port sustainability management based on a Bayesian network model Application to \\ the spanish port system
}

Beatriz Molina Serrano $^{1 *} \quad$ Nicoletta González-Cancelas ${ }^{1} \quad$ Francisco Soler-Flores $^{2}$

Recibido 14 de marzo de 2017, aceptado 25 de septiembre de 2017

Received: March 14, 2017 Accepted: September 25, 2017

\begin{abstract}
RESUMEN
En la explotación y gestión portuaria entran en juego numerosas variables, necesitando el gestor de la infraestructura conocer las relaciones entre ellas para poder modificar condiciones de la explotación. Con el empleo de redes bayesianas se pueden clasificar, predecir y diagnosticar dichas variables, al permitir estimar la probabilidad posterior de las variables no conocidas en base a las variables conocidas. Esto quiere decir, a nivel de planificación, que no se tienen que conocer todas las variables, al conocer sus relaciones. Por ello, estas redes pueden utilizarse para tomar decisiones óptimas introduciendo posibles acciones y la utilidad de sus resultados.

En la metodología que se propone se ha generado una base de datos con más de 100 variables portuarias, clasificadas en económicas, sociales, ambientales e institucionales, tal como se abordan los estudios de smart ports, para el Sistema Portuario Español. A partir de ella se ha generado una red usando un grafo dirigido acíclico, que permite conocer las relaciones entre las variables portuarias en términos de padres e hijos, modelando la incertidumbre de forma probabilística incluso cuando el número de variables es elevado, como ocurre en la explotación y planificación portuaria.

La principal conclusión extraída es que las variables económicas son la causa del resto de tipologías de variables y ejercen el papel de padres de la red en la mayoría de los casos. Por otro lado, también se concluye que, conocidas variables de tipo ambiental, la red permite estimar la probabilidad posterior de las variables sociales.
\end{abstract}

Palabras clave: Variables portuarias, sostenibilidad, gestión portuaria, redes bayesianas, sistema portuario español.

\begin{abstract}
Numerous variables are involved in port operation and management, and infrastructure managers need to know relationships between them, to be able to modify operating conditions. Using Bayesian networks makes possible to classify, predict and diagnose these variables, allowing to estimate the posterior probability of the unknown variables based on the known variables. This means, at the planning level, that it is not necessary to know all the variables, knowing their relationships. Therefore, these networks can be used to make optimal decisions introducing possible actions and usefulness of their results.

In the methodology proposed, a database with more than 100 port variables has been generated. Variables are classified as economic, social, environmental and institutional, as it is made in smart port studies in all Spanish Port System. Using this database, a network has been generated using an acyclic directed graph, which allows knowing relations between port variables regarding parents and sons. This kind of network allows modeling uncertainty probabilistically even when the number of variables is high as in the case of port planning and exploitation.
\end{abstract}

1 Departamento de Ingeniería Civil. Transportes. Universidad Politécnica de Madrid. Avda. Profesor Aranguren s/n 28040 Madrid (España).E-mail: beatriz.molinas@alumnos.upm.es; nicoleta.gcancelas@upm.es

2 Departamento de Matemáticas e Informática aplicadas a la Ingeniería Civil y Naval. Universidad Politécnica de Madrid. Avda. Profesor Aranguren s/n 28040 Madrid (España).E-mail: f.soler@upm.es

* Autor de correspondencia 
The main conclusion of the study is that economic variables are the cause of the other typologies and they play the role of parents in the network in most of the cases. Moreover, another conclusion is related to environmental variables. So, if they are known, the network will allow estimating the subsequent probability of social ones.

Keywords: Port variables, sustainability, port management, bayesian networks, spanish port system.

\section{INTRODUCCIÓN}

El concepto de desarrollo sostenible partió de la búsqueda de compatibilidad entre el desarrollo económico y la protección y uso adecuado de los recursos naturales. Está visión fue ampliándose a otras dimensiones, de forma que actualmente, el concepto de desarrollo sostenible posee un carácter integral, multidimensional e interactivo. Este concepto de sostenibilidad está siendo aplicado en forma emergente por autoridades del sector transporte y muchos otros campos de actividad e industrias a nivel mundial, fuertemente impulsada por iniciativas que incorporan la variable ambiental y la responsabilidad social empresarial en la gestión estratégica de las empresas [1]. En el caso de los puertos, la sostenibilidad portuaria tiene sus raíces en las propuestas del GRI [2] de las cuáles conserva, entre otras cuestiones, los cuatro ejes o dimensiones que conforman un enfoque de desarrollo sostenible, es decir, el institucional, el económico, el ambiental y el social (Figura 1).

Fuente: Elaboración propia a partir de [1].

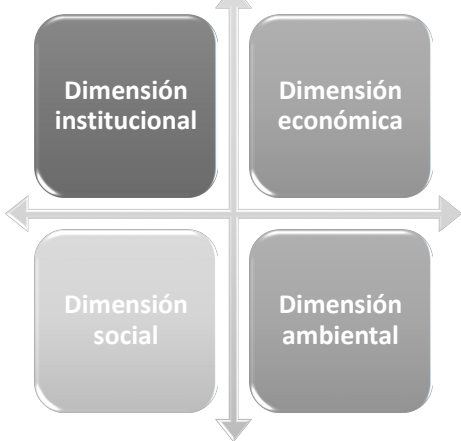

Figura 1. Cuatro dimensiones de la sostenibilidad portuaria.

De esta forma se considera que la gestión sostenible de una empresa u organismo tiene como meta el mantenimiento equilibrado en el largo plazo de su función $\mathrm{y}$ actividad, de modo que se tengan en cuenta el efecto reciproco de su actividad con el entorno económico, social y ambiental con el que se relaciona y el efecto de su actividad con su propia estructura al tiempo que se el efecto de sus funciones y actividades en sus aspectos o dimensiones económica, social, medioambiental e institucional, buscando un desarrollo equilibrado de estas cuatro dimensiones [3].
La búsqueda del desarrollo sostenible ha creado una nueva disciplina científica, llamada Ciencia de la Sostenibilidad, con problemas que no pueden enfrentarse con las herramientas metodológicas de las disciplinas científicas clásicas. Esta disciplina explora las relaciones e interacciones de la actividad humana con los ecosistemas que soportan la vida para encontrar un camino hacia un desarrollo humano sostenible [4].

En este contexto, merece una atención particular el transporte marítimo, al transportar alrededor del $80 \%$ del volumen del comercio internacional (en toneladaskilómetros) a nivel mundial [5]. Así, en el sector portuario, se debe entender la gestión sostenible como "aquella que permite que crezca el volumen de tráfico de contenedores, graneles sólidos y líquidos, mercancía general y número de pasajeros, disminuyendo a su vez el consumo de energía y recursos naturales, el volumen de residuos generados y los impactos negativos a los sistemas sociales y ecosistemas en las áreas de influencia del puertos" [6].

Sin embargo, uno de los principales desafíos que plantea la introducción de criterios de sostenibilidad en el modelo de gestión y desarrollo portuario es romper la inercia existente en relación a la consideración "única" del factor económico como variable de desarrollo, y lograr que las variables ambiental y social tomen la importancia que deben a fin de que el modelo de gestión y desarrollo portuario realmente tienda hacia la sostenibilidad [7].

El Texto Refundido de la Ley de Puertos del Estado y de la Marina Mercante (BOE núm. 253, de 20/10/2011), incorpora la sostenibilidad como uno de los principios que deben regir el modelo de planificación y de gestión de los puertos. Para ello, en el artículo 55.4 prevé que el proyecto de Plan de Empresa de cada Autoridad Portuaria debe acompañarse de una Memoria de Sostenibilidad, la cual constituye una herramienta de análisis y diagnóstico. Dicha memoria se lleva a cabo a través de una metodología, basada en el GRI a la cual se incorpora la constitución de indicadores específicos, y es aprobada por Puertos del Estado y las Autoridades Portuarias. La redacción de estas Memorias de Sostenibilidad, supone un esfuerzo de integración de información sobre el comportamiento de la Autoridad Portuaria y su desempeño ambiental, económico 
y social, si bien no determinan el comportamiento de la misma, sino que describe, por medio de indicadores de desempeño, los resultados derivados de la adopción y aplicación de códigos, políticas y sistemas de gestión [8]. Estos indicadores de sostenibilidad permiten evaluar el desempeño de la gestión de desarrollo sostenible, más allá de los reportes de sostenibilidad, los indicadores permiten controlar de manera objetiva la marcha de la gestión de la Autoridad Portuaria en esta materia. Por ello, resulta obvio que los indicadores deben cubrir las cuatro dimensiones de la sostenibilidad. La aplicación de los indicadores es útil para que las Autoridades Portuarias controlen su gestión sostenible, evalúen el impacto de los programas aplicados y los modifiquen cuando sea necesario. Los indicadores permiten realizar Benchmarking de gestión sostenible entre puertos para determinar las mejores prácticas y comparar el desempeño de una Autoridad Portuaria frente a la industria similar, $y$ en el marketing puede ser un elemento diferenciador y de competitividad en el mercado. La aplicación generalizada a un sistema portuario permitiría realizar un benchmarking preciso en materia de sostenibilidad entre puertos de una misma región o país [9].

De la aplicación de dichas herramientas surgen los objetivos (económicos, medio ambientales, sociales e institucionales) que una autoridad o empresa portuaria debe alcanzar para asegurar el desarrollo sostenible y crecimiento de su puerto [10]. Los objetivos económicos pueden ser: el incremento del volumen de negocio, aumentar los ingresos por concesiones, reducir el endeudamiento con el fin de asegurar la sostenibilidad financiera del puerto y optimizar y rentabilizar las inversiones de los activos portuarios. En el caso de la dimensión medio ambiental, los objetivos pueden ser tales como accionar con respeto al medio ambiente, minimizar los impactos ambientales derivados de la actividad portuaria, minimizar los accidentes ambientales y mejorar la gestión ambiental en el recinto portuario. Los objetivos sociales que pueden ser internos y externos, deben estar enmarcados en ámbitos tales como desarrollar y modernizar sistemas de gestión de los recursos humanos, desarrollar un equipo humano motivado y comprometido y lograr un respaldo sostenido y activo de la comunidad del entorno. Para los objetivos institucionales se puede estar buscando impulsar ciertos cambios legales y normativos para modernizar la forma de desarrollo y operación del puerto, reorganizar el mercado portuario incorporando competencia, gestión e inversión privada para mejor su eficiencia y capacidad de expansión, modernizar el régimen laboral para mejorar su competitividad, desarrollar la comunidad portuaria para incrementar la eficiencia operacional y calidad de los procesos, institucionalizar y optimizar la relación ciudad puerto, expandir la gestión operativa del puerto a la cadena logística para agregar valor e integrar a la comunidad logística local al desarrollo del puerto (Figura2).

Fuente: Elaboración propia a partir de [1].

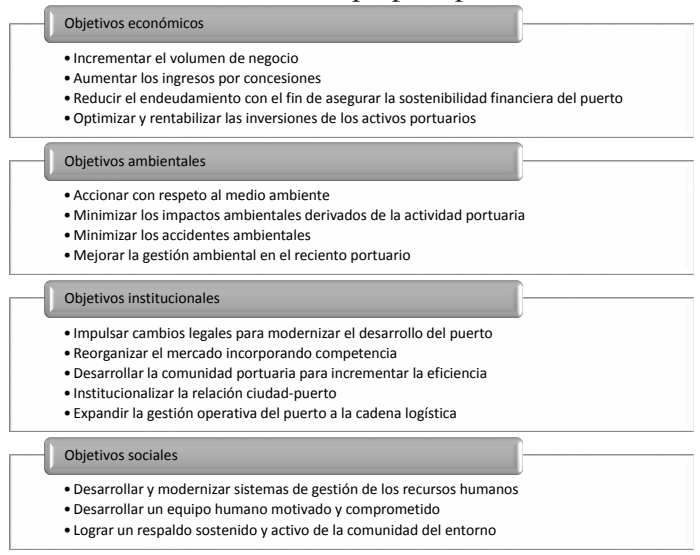

Figura 2. Objetivos estratégicos para las cuatro dimensiones de la sostenibilidad.

Por tanto, el objetivo que se persigue es que través de estos cuatro ejes de la sostenibilidad, los puertos se conformen como un sistema y no sean vistos como entes aislados y sujetos a una coyuntura comercial concreta, sino como elementos que se interrelacionan con un entorno físico, social y ambiental, en el que han de integrarse de forma efectiva, esto es, siendo capaces de adaptarse a una coyuntura cambiante y a la vez, apuntando a una renovación que contribuya a alcanzar el mejor de los escenarios futuros posibles [11].

Sin embargo, una fuerte limitación metodológica no ha permitido una más extensa aplicación del concepto. Aún es una cuestión crítica y no resuelta para la gestión del desarrollo sostenible, la disponibilidad de metodologías que permitan evaluar el impacto del accionar de las instituciones y empresas en cada una de las dimensiones de la sostenibilidad, determinando el valor y las variables que cuantifiquen la verdadera contribución o aporte de esa gestión al desarrollo sostenible.

Una de las metodologías a emplear son las Redes Bayesianas, a partir de las cuales se puede obtener de una forma gráfica las relaciones entre las variables consideradas de cada una de las cuatro dimensiones, con objeto de poder determinar a posteriori los valores que cuantifiquen su contribución a la sostenibilidad. 
En la tabla siguiente (Tabla 1), se incluyen los principales trabajos sobre sistemas de transportes que han sido desarrollados usando Redes Bayesianas:

Tabla 1. Principales trabajos sobre sistemas de transportes que han sido desarrollados usando Redes Bayesianas.

\begin{tabular}{|c|c|c|}
\hline Autores & Año & Título \\
\hline $\begin{array}{c}\text { Friedman, N., \& } \\
\text { Goldszmidt, M. [12] }\end{array}$ & 1996 & $\begin{array}{c}\text { Building classifiers using } \\
\text { Bayesian networks }\end{array}$ \\
\hline $\begin{array}{c}\text { Jara-Díaz, S., } \\
\text { Martínez-Budría, E., } \\
\text { Cortes, C., \& Vargas, } \\
\text { A. [13] }\end{array}$ & 1997 & $\begin{array}{l}\text { Marginal costs and scale } \\
\text { economies in Spanish ports }\end{array}$ \\
\hline $\begin{array}{l}\text { Tebaldi, C., \& West, } \\
\text { M. [14] }\end{array}$ & 1998 & $\begin{array}{l}\text { Bayesian inference on network } \\
\text { traffic using link count data }\end{array}$ \\
\hline Cain, J. [15] & 2001 & $\begin{array}{l}\text { Planning improvements in } \\
\text { natural resource management. } \\
\text { guidelines for using Bayesian } \\
\text { networks to support the } \\
\text { planning and management of } \\
\text { development programmes in } \\
\text { the water sector and beyond }\end{array}$ \\
\hline $\begin{array}{c}\text { Conati, C., Gertner, } \\
\text { A. y VanLehn, K. } \\
{[16]}\end{array}$ & 2002 & $\begin{array}{l}\text { Using Bayesian networks to } \\
\text { manage uncertainty in student } \\
\text { modeling. User Modeling and } \\
\text { User-Adapted Interaction }\end{array}$ \\
\hline $\begin{array}{l}\text { Bromley, J., Jackson, } \\
\text { N. A., Clymer, O. J., } \\
\text { Giacomello, A. M., } \\
\text { \& Jensen, F. V. [17] }\end{array}$ & 2005 & $\begin{array}{l}\text { The use of hugin } ® \text { to develop } \\
\text { Bayesian networks as an aid } \\
\text { to integrated water resource } \\
\text { planning }\end{array}$ \\
\hline $\begin{array}{c}\text { Sun, S., Zhang, C., \& } \\
\text { Yu, G. [18] }\end{array}$ & 2006 & $\begin{array}{l}\text { A Bayesian network approach } \\
\text { to traffic flow forecasting }\end{array}$ \\
\hline $\begin{array}{l}\text { Zheng, W., Lee, D. } \\
\text { H., \& Shi, Q. [19] }\end{array}$ & 2006 & $\begin{array}{l}\text { Short-term freeway traffic flow } \\
\text { prediction: Bayesian combined } \\
\text { neural network approach }\end{array}$ \\
\hline $\begin{array}{c}\text { Janssens, D., } \\
\text { Wets, G., Brijs, } \\
\text { T., Vanhoof, K., } \\
\text { Arentze, T., \& } \\
\text { Timmermans, H. [20] }\end{array}$ & 2006 & $\begin{array}{l}\text { Integrating Bayesian } \\
\text { networks and decision trees } \\
\text { in a sequential rule-based } \\
\text { transportation model }\end{array}$ \\
\hline $\begin{array}{c}\text { Castillo, E., } \\
\text { Menéndez, J. M., } \\
\text { and Sánchez- } \\
\text { Cambronero, S. [21] }\end{array}$ & 2008 & $\begin{array}{c}\text { Traffic estimation and optimal } \\
\text { counting location without path } \\
\text { enumeration using Bayesian } \\
\text { networks }\end{array}$ \\
\hline $\begin{array}{c}\text { Castillo, E., } \\
\text { Menéndez, J. } \\
\text { M., \& Sánchez- } \\
\text { Cambronero, S. [22] }\end{array}$ & 2008 & $\begin{array}{l}\text { Predicting traffic flow using } \\
\text { Bayesian networks }\end{array}$ \\
\hline $\begin{array}{l}\text { Trucco, P., Cagno, } \\
\text { E., Ruggeri, F., \& } \\
\text { Grande, O. [23] }\end{array}$ & 2008 & $\begin{array}{c}\text { A Bayesian Belief Network } \\
\text { modelling of organisational } \\
\text { factors in risk analysis: } \\
\text { A case study in maritime } \\
\text { transportation }\end{array}$ \\
\hline $\begin{array}{l}\text { Klemola, E., } \\
\text { Kuronen, J., Kalli, J., } \\
\text { Arola, T., Hanninen, } \\
\text { M., Lehikoinen, A., } \\
\text { \& Tapaninen, U. [24] }\end{array}$ & 2009 & $\begin{array}{l}\text { A cross-disciplinary approach } \\
\text { to minimising the risks of } \\
\text { maritime transport in the Gulf } \\
\text { of Finland. }\end{array}$ \\
\hline $\begin{array}{l}\text { Kaluza, P., Kölzsch, } \\
\text { A., Gastner, M. T., \& } \\
\text { Blasius, B. [25] }\end{array}$ & 2010 & $\begin{array}{l}\text { The complex network of } \\
\text { global cargo ship movements. }\end{array}$ \\
\hline $\begin{array}{l}\text { Hofleitner, A., } \\
\text { Herring, R., Abbeel, } \\
\text { P., \& Bayen, A. [26] }\end{array}$ & 2012 & $\begin{array}{l}\text { Learning the dynamics of } \\
\text { arterial traffic from probe data } \\
\text { using a dynamic Bayesian } \\
\text { network }\end{array}$ \\
\hline
\end{tabular}

\begin{tabular}{|c|c|c|}
\hline Autores & Año & Título \\
\hline $\begin{array}{l}\text { Cancelas, N. G., } \\
\text { Flores, F. S., \& } \\
\text { Orive, A. C. [27] }\end{array}$ & 2013 & $\begin{array}{l}\text { Modelo de eficiencia de las } \\
\text { terminales de contenedores del } \\
\text { sistema portuario español }\end{array}$ \\
\hline $\begin{array}{l}\text { Camarero, A., } \\
\text { González-Cancelas, } \\
\text { N., Soler, F., \& } \\
\text { López, I. [28] }\end{array}$ & 2013 & $\begin{array}{c}\text { Utilización de redes } \\
\text { bayesianas como método de } \\
\text { caracterización de parámetros } \\
\text { físicos de las terminales de } \\
\text { contenedores del sistema } \\
\text { portuario español }\end{array}$ \\
\hline $\begin{array}{l}\text { Flores, F. S., Cancelas, } \\
\text { N. G., Orive, A. C., } \\
\text { Gárate, J. L. A., \& } \\
\text { Monzón [29] }\end{array}$ & 2014 & $\begin{array}{c}\text { Diseño de un modelo de } \\
\text { planificación de zonas de } \\
\text { actividades logísticas mediante } \\
\text { el empleo de redes bayesianas }\end{array}$ \\
\hline $\begin{array}{l}\text { Li, K. X., Yin, J., } \\
\text { Bang, H. S., Yang, } \\
\text { Z., \& Wang, J. [30] }\end{array}$ & 2014 & $\begin{array}{c}\text { Bayesian network with } \\
\text { quantitative input for maritime } \\
\text { risk analysis }\end{array}$ \\
\hline $\begin{array}{c}\text { Rodríguez, T., } \\
\text { González Cancelas, } \\
\text { N., \& Soler-Flores, } \\
\text { F [31] }\end{array}$ & 2015 & $\begin{array}{l}\text { Setting the Port Planning } \\
\text { Parameters In Container } \\
\text { Terminals through Bayesian } \\
\text { Networks }\end{array}$ \\
\hline $\begin{array}{c}\text { Rodríguez García, } \\
\text { T. [32] }\end{array}$ & 2016 & $\begin{array}{l}\text { Aplicaciones tecnológicas } \\
\text { en la logística de transportes } \\
\text { portuarios. }\end{array}$ \\
\hline
\end{tabular}

\section{METODOLOGÍA}

Para la consecución del objetivo de caracterizar los parámetros físicos de las terminales de contenedores del sistema portuario español, mediante redes bayesianas, se ha desarrollado la siguiente metodología (Figura 3). Esta se divide en dos tareas: una para determinar el escenario de trabajo y la segunda para desarrollar el modelo de inteligencia artificial.

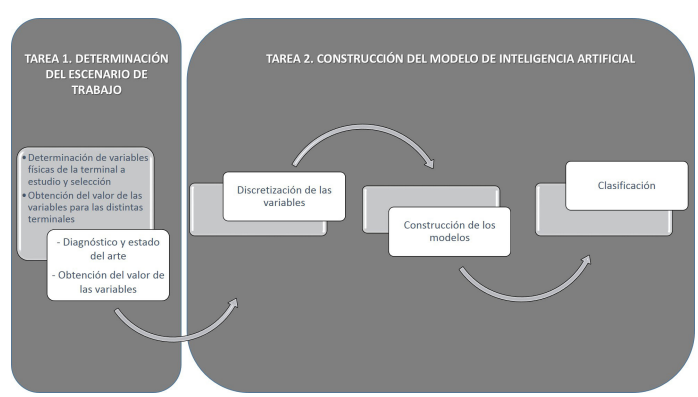

Figura 3. Esquema de la metodología empleada.

Las redes bayesianas están diseñadas para hallar las relaciones de dependencia e independencia entre todas las variables que conforman el dominio de estudio. Basado en ello, se utilizan métodos de razonamiento probabilístico que permiten realizar predicciones sobre el valor de cualquier variable desconocida basados en los valores de las conocidas. 
Las redes bayesianas proveen una forma compacta de representar el conocimiento y métodos flexibles de razonamiento -basados en las teorías probabilísticascapaces de predecir el valor de variables no observadas y explicar las observadas. Entre las características que poseen las redes bayesianas se puede destacar que permiten aprender sobre relaciones de dependencia y causalidad, permiten combinar conocimiento con datos y pueden manejar bases de datos incompletas.

Las redes bayesianas pueden realizar la tarea de clasificación -caso particular de predicción- que se caracteriza por tener una sola de las variables de la base de datos (clasificador) que se desea predecir, mientras que todas las otras son los datos propios del caso que se desea clasificar. Pueden existir una gran cantidad de variables en la base de datos, algunas de las cuales estén directamente relacionadas con la variable clasificadora pero también otras variables que tienen una influencia directa sobre dicha clase.

El obtener una red bayesiana a partir de datos es un proceso de aprendizaje que se divide en dos etapas: el aprendizaje estructural y el aprendizaje paramétrico [33]. La primera de ellas consiste en obtener la estructura de la red bayesiana, es decir, las relaciones de dependencia e independencia entre las variables involucradas. La segunda etapa tiene como finalidad obtener las probabilidades a priori y condicionales requeridas a partir de una estructura dada.

\section{Tarea 1: determinación del escenario de trabajo}

\section{Diagnóstico y estado del arte}

Consiste en la revisión del estado del arte para identificar el conjunto de variables de medida de la sostenibilidad, mediante el empleo de buscadores especializados y gestores de aplicaciones.

Determinación de las variables físicas de la terminal y selección

Se realiza un estudio las variables de sostenibilidad susceptibles de investigación para los puertos marítimos. Dada la variabilidad de las terminales y puertos componen el sistema portuario español se debe recurrir a las memorias de sostenibilidad de las autoridades portuarias del sistema portuario español.

\section{Obtención del valor de las variables}

El Sistema Portuario español de titularidad estatal está integrado por 46 puertos de interés general, gestionados por 28 Autoridades Portuarias, cuya coordinación y control de eficiencia corresponde al Organismo Público Puertos del Estado, órgano dependiente del Ministerio de Fomento y que tiene atribuida la ejecución de la política portuaria del Gobierno. (www.puertos. es/es-es). Los valores de las variables con las que se trabaja para la construcción del modelo de la red bayesiana corresponden a los datos de las Memorias de Sostenibilidad publicadas anualmente por las Autoridades Portuarias de los puertos españoles y completados con información suministrada por el Organismo Público Puertos del Estado, corresponden al registro histórico desde el año 2010, contando con casi 3000 registros.

A través de las memorias de sostenibilidad, Puertos del Estado y las Autoridades Portuarias materializan su compromiso con la transparencia en su gestión; proporcionando una visión amplia de sus logros y retos en aspectos como la competitividad, la calidad en la prestación de servicios, la eficiencia en el uso de recursos y su impacto sobre su entorno, económico, social y natural. La Memoria se divide en cuatro dimensiones: institucional, económica, ambiental y social, de acuerdo también a la Guía para la elaboración de las memorias de sostenibilidad de las autoridades portuarias aprobada por Puertos del Estado. En cada apartado se describe el enfoque que las Autoridades Portuarias desarrollan para su adecuada gestión y se informa sobre los resultados conseguidos, mediante los correspondientes indicadores.

Las variables seleccionadas en el estudio se incluyen en la Tabla 2, la cual se muestra a continuación:

Tabla 2. Relación de variables empleadas.

\begin{tabular}{|c|c|}
\hline $\begin{array}{c}\text { Dimensión } \\
\text { Institucional (ID: } \\
\text { dimins) }\end{array}$ & $\begin{array}{c}\text { Conjunto de objetivos e indicadores de } \\
\text { sostenibilidad que reflejan prioridades } \\
\text { estratégicas en el ámbito de la } \\
\text { sostenibilidad para el conjunto del } \\
\text { sistema portuario de interés general }\end{array}$ \\
\hline Marca temporal & Descripción \\
\hline $\begin{array}{c}\text { Herramientas de } \\
\text { apoyo a la gestión } \\
\text { (ID: herramgestion_ } \\
\text { dimins) }\end{array}$ & $\begin{array}{c}\text { Sistemas de gestión de apoyo a la toma } \\
\text { de decisiones: sistemas de gestión de } \\
\text { la calidad, cuadros de mando integral, } \\
\text { campañas de caracterización de } \\
\text { mercados, etc. }\end{array}$ \\
\hline $\begin{array}{c}\text { Generación de } \\
\text { infraestructura portuaria } \\
\text { (ID: geninfraortuaria_ } \\
\text { dimins) }\end{array}$ & $\begin{array}{c}\text { Papel de la Autoridad Portuaria como } \\
\text { proveedor de la infraestructura }\end{array}$ \\
\hline
\end{tabular}




\begin{tabular}{|c|c|}
\hline $\begin{array}{l}\text { Mercados servidos (ID: } \\
\text { mercservidos_dimins) }\end{array}$ & $\begin{array}{l}\text { Estructura y evolución de los } \\
\text { principales tráficos de mercancías }\end{array}$ \\
\hline $\begin{array}{l}\text { Papel del sector } \\
\text { portuario como } \\
\text { dinamizador de la } \\
\text { actividad productiva } \\
\text { (ID: dinamact_dimins) }\end{array}$ & $\begin{array}{l}\text { Principales sectores o actividades } \\
\text { relevantes en el desarrollo económico } \\
\text { local que se apoyan en el puerto para } \\
\text { su desarrollo }\end{array}$ \\
\hline $\begin{array}{l}\text { Servicios y concesiones/ } \\
\text { autorizaciones (ID: } \\
\text { serviciosconcauto_ } \\
\text { dimins) }\end{array}$ & $\begin{array}{l}\text { Tipos, marco de prestación y } \\
\text { regulación }\end{array}$ \\
\hline $\begin{array}{l}\text { Presencia de la } \\
\text { iniciativa privada (ID: } \\
\text { inicprivada_dimins) }\end{array}$ & $\begin{array}{l}\text { Número de empresas que operan en } \\
\text { el puerto, superficie terrestre ocupada, } \\
\text { caracterizada como uso comercial } \\
\text { concesionado, etc. }\end{array}$ \\
\hline $\begin{array}{l}\text { Transparencia y libre } \\
\text { concurrencia (ID: } \\
\text { transconcu_dimins) }\end{array}$ & $\begin{array}{l}\text { Iniciativas dirigidas a garantizar que todo } \\
\text { operador que desee prestar servicios } \\
\text { en el puerto u optar a una concesión } \\
\text { pueda conocer de modo transparente las } \\
\text { condiciones para operar en el puerto y los } \\
\text { mecanismos administrativos que regulan } \\
\text { dicho proceso }\end{array}$ \\
\hline $\begin{array}{l}\text { Calidad en la } \\
\text { prestación de los } \\
\text { servicios (ID: } \\
\text { calidserv_dimins) }\end{array}$ & $\begin{array}{l}\text { Iniciativas promovidas por la Autoridad } \\
\text { Portuaria dirigidas a mejorar la } \\
\text { eficiencia, la calidad del servicio y el } \\
\text { rendimiento de los servicios prestados } \\
\text { a la mercancía }\end{array}$ \\
\hline $\begin{array}{l}\text { Integración de los } \\
\text { puertos en el sistema } \\
\text { de transporte (ID: } \\
\text { intetrans_dimins) }\end{array}$ & $\begin{array}{c}\text { Eficiencias con la que son coordinados } \\
\text { los diferentes modos de transporte que } \\
\text { confluyen en el puerto }\end{array}$ \\
\hline $\begin{array}{l}\text { Dimensión económica } \\
\text { (ID: dimecon) }\end{array}$ & $\begin{array}{l}\text { Situación económico financiera del } \\
\text { sistema portuario español }\end{array}$ \\
\hline Marca temporal & Descripción \\
\hline $\begin{array}{l}\text { Situación económica } \\
\text { financiera (ID: } \\
\text { sitecofin_dimecon) }\end{array}$ & $\begin{array}{l}\text { Entre otros indicadores: rentabilidad sobre } \\
\text { activos, EBIDTA/tonelada, servicio de la } \\
\text { deuda, relación gastos de explotación e } \\
\text { ingresos de explotación, etc. }\end{array}$ \\
\hline $\begin{array}{l}\text { Nivel y estructura de } \\
\text { las inversiones (ID: } \\
\text { inv_dimecon) }\end{array}$ & $\begin{array}{l}\text { Entre otros indicadores: inversión } \\
\text { pública en relación con el cash-flow, } \\
\text { inversión ajena frente a la inversión } \\
\text { pública, renovación de activos }\end{array}$ \\
\hline $\begin{array}{l}\text { Negocio y servicios } \\
\text { (ID: negserv_dimecon) }\end{array}$ & $\begin{array}{c}\text { Entre otros indicadores: ingresos por } \\
\text { tasas de ocupación y actividad, uso } \\
\text { comercial de la superficie, uso de los } \\
\text { muelles, etc. }\end{array}$ \\
\hline $\begin{array}{l}\text { Valor generado y } \\
\text { productividad (ID: } \\
\text { vgenprod_dimecon) }\end{array}$ & $\begin{array}{c}\text { Entre otros indicadores: productividad } \\
\text { del trabajo según ingresos, } \\
\text { productividad del trabajo según } \\
\text { EBIDTA, etc. }\end{array}$ \\
\hline $\begin{array}{c}\text { Dimensión social (ID: } \\
\text { dimsoci) }\end{array}$ & Capital humano y empleo \\
\hline Marca temporal & Descripción \\
\hline $\begin{array}{l}\text { Capital humano de } \\
\text { la actividad portuaria } \\
\text { (ID: caphum_dimsoci) }\end{array}$ & $\begin{array}{l}\text { Empleo, comunicación interna y } \\
\text { participación, formación, estructura de } \\
\text { plantilla y equidad, seguridad y salud } \\
\text { en el trabajo, etc. }\end{array}$ \\
\hline $\begin{array}{l}\text { Empleo y seguridad } \\
\text { laboral en la comunidad } \\
\text { portuaria (ID: empl_ } \\
\quad \text { dimsoci) }\end{array}$ & $\begin{array}{l}\text { Empleo en la comunidad portuaria, } \\
\text { seguridad laboral y formación en } \\
\text { servicios y concesiones portuarios, etc. }\end{array}$ \\
\hline
\end{tabular}

\begin{tabular}{|c|c|}
\hline $\begin{array}{c}\text { Dimensión } \\
\text { medioambiental (ID: } \\
\text { dimma) }\end{array}$ & Calidad y gestión ambiental \\
\hline Marca temporal & Descripción \\
\hline $\begin{array}{l}\text { Gestión ambiental } \\
\text { (ID: gestamb_dimma) }\end{array}$ & $\begin{array}{l}\text { Grados de implantación de los sistemas } \\
\text { de gestión ambiental (EMAS, ISO } \\
14001 \text { y PERLS) y recursos económicos } \\
\text { invertidos gastos, así como inversiones } \\
\text { en su caso, asociados a la implantación, } \\
\text { certifiación y mantenimiento de un } \\
\text { sistema de gestión ambiental }\end{array}$ \\
\hline $\begin{array}{l}\text { Calidad del aire (ID: } \\
\text { calaire_dimma) }\end{array}$ & $\begin{array}{l}\text { Principales focos de emisión del puerto } \\
\text { que suponen emisiones significativas, } \\
\text { evolución del número de quejas o } \\
\text { denuncias registradas por la Autoridad } \\
\text { Portuarias procedentes de grupos de } \\
\text { interés realtivas a emisiones de polvo o } \\
\text { a la calidad del aire en general, medidas } \\
\text { implantadas por la Autoridad Portuaria } \\
\text { para controlar las emisiones ligadas a la } \\
\text { actividad del conjunto del puerto }\end{array}$ \\
\hline $\begin{array}{l}\text { Calidad del agua (ID: } \\
\text { calagua_dimma) }\end{array}$ & $\begin{array}{c}\text { Principales focos de vertido situados en el } \\
\text { puerto que tienen un impacto significativo } \\
\text { en la calidad del agua y sedimentos de las } \\
\text { dársenas del puerto, medidas implantadas } \\
\text { por la Autoridad Portuaria para controlar } \\
\text { las emisiones ligadas a la actividad del } \\
\text { conjunto del puerto, superficie de la zona } \\
\text { de servicio que cuenta con recogida y } \\
\text { tratamiento de aguas residuales }\end{array}$ \\
\hline $\begin{array}{l}\text { Calidad acústica (ID: } \\
\text { calacust_dimma) }\end{array}$ & $\begin{array}{l}\text { Principales focos de emisión (puntuales } \\
\text { y difusos) del puerto que suponen } \\
\text { emisiones acústicas significativas, } \\
\text { evolución del número de quejas o } \\
\text { denuncias registradas por la Autoridad } \\
\text { Portuaria procedentes de grupos de } \\
\text { interés, elaboración de mapa de ruido y } \\
\text { plan de acción acústica }\end{array}$ \\
\hline $\begin{array}{l}\text { Gestión de residuos } \\
\text { (ID: residuos_dimma) }\end{array}$ & $\begin{array}{l}\text { Residuos generados por la Autoridad } \\
\text { Portuaria que son segregados y } \\
\text { valorizados, actividades o fuentes de } \\
\text { generación de residuos dentro del puerto, } \\
\text { iniciativas promovidas por la Autoridad } \\
\text { Portuaria para la mejora de la gestión de } \\
\text { residuos de la comunidad portuaria }\end{array}$ \\
\hline $\begin{array}{l}\text { Ecoeficiencia (ID: } \\
\text { ecoef_dimma) }\end{array}$ & $\begin{array}{c}\text { Eficiencia en el uso del suelo, consumo } \\
\text { de agua y energía eléctrica por la } \\
\text { Autoridad Portuaria }\end{array}$ \\
\hline $\begin{array}{l}\text { Comunidad portuaria } \\
\text { (ID: comport_dimma) }\end{array}$ & $\begin{array}{l}\text { Condiciones o exigencias sobre } \\
\text { aspectos ambientales en los pliegos de } \\
\text { prescripciones técnicas particulares de } \\
\text { los servicios portuarios, en términos de } \\
\text { otorgamiento y en títulos de concesión } \\
\text { o autorización }\end{array}$ \\
\hline
\end{tabular}

Sin embargo, los modelos directos tienen una noción más compleja de independencia que los modelos indirectos, pero tienen, en cambio, varias ventajas. La ventaja principal de estos modelos es que se puede crear un arco desde A hasta B para indicar que A es la 
"causa" de B, lo cual se puede usar para crear la estructura de grafo. Es por ello que los modelos directos pueden codificar relaciones determinísticas, siendo más fáciles de aprender (encajar los datos).

Asimismo, es necesario especificar los parámetros del modelo para así poder definir la estructura del grafo. En el caso de modelos directos de Distribución Condicional de la Probabilidad (CPD), dicha definición se debe especificar para cada uno de los nodos. En el caso de que las variables sean discretas, se deben representar como en la tabla (CPT), es decir, definiendo la probabilidad que un nodo hijo tiene para cada uno de los diferentes valores para cada una de las combinaciones de valores de sus nodos padres.

\section{Tarea 2: construcción del modelo de inteligencia artificial}

Los modelos gráficos de probabilidad son grafos en los cuales los nodos representan variables aleatorias y en los que la existencia o la falta de arcos representan supuestos de independencia condicional. Por lo tanto, proporcionan una representación completa de la articulación de las distribuciones de la probabilidad. Los modelos gráficos indirectos, también llamados Campos aleatorios de Markov (MRFs) o Redes de Markov, cuenta con una definición simple de independencia: dos nodos o conjunto de nodos, A y B, son condicionalmente independientes considerando un tercer conjunto $(\mathrm{C})$, si todos las caminos existentes entre los nodos A y B están separados por un nodo en C. Por el contrario, los modelos gráficos directos, también llamados Redes Bayesianas o Redes de Creencias (BNs), cuentan con una noción más compleja de independencia, la cual tiene en cuenta la direccionalidad de los arcos, tal y como se explica a continuación [34].

Los modelos gráficos de tipo indirectos son más populares en el caso de comunidades físicas y de visión, mientras que los modelos directos lo con en comunidades AI y estadísticas. No obstante, es posible tener un modelo en el cual se incluyen arcos directos e indirectos, denominándose a este modelo como Grafo encadenado. Para realizar un estudio más exhaustivo de las relaciones entre los modelos gráficos directos e indirectos, revisar las siguientes referencias: [35-37].

Con objeto de especificar la Red Bayesiana y así representar totalmente las uniones en la distribución de la probabilidad, se necesita definir la distribución de la probabilidad condicionada de $\mathrm{X}$ hacia sus padres en cada uno de los nodos X. La distribución condicional de X hacia sus padres puede adoptar cualquier forma. Es muy común el uso de distribuciones discretas o Gaussianas cuando esto simplifica los cálculos. Otras veces, sólo se conocen las restricciones de la distribución, en cuyo caso, se puede usar el máximo principio de la entropía para definir una distribución sencilla: tomar el que tiene una mayor entropía de las restricciones. De forma análoga, en el contexto específico de una Red Bayesiana dinámica, es habitual que se especifique la distribución condicional del estado oculto de la evolución temporal para maximizar el ratio de proceso estocástico implícito.

A menudo, estas distribuciones condicionales incluyen parámetros que son desconocidos y deben ser estimados a partir de los datos. Algunas veces, los parámetros se estiman usando el enfoque de máxima verosimilitud. La maximización de la verosimilitud (o probabilidad posterior) de forma directa resulta generalmente complejo cuando existen variables no observables. Un enfoque típico de este problema es el algoritmo de expectación-maximización. Este algoritmo alterna el cálculo de los valores esperados de las variables no observadas, que se determinan a partir de los datos observados, y la maximización de la verosimilitud completa (o posterior), considerando que los valores esperados calculados previamente son correctos. Bajo condiciones de regularidad suave, este proceso converge en valores de parámetro de máxima verosimilitud (o máximo posterior).

Un enfoque más profundo de los parámetros bayesianos es tratar a los parámetros como variables no observadas adicionales y computarlas en una distribución completa posterior con todos los nodos condicionales sobre los datos observados, con objeto de sacar los parámetros. Este enfoque puede ser costoso y conducir a modelos de gran dimensión, por lo que en la práctica es más común el uso de métodos clásicos de parametrización.

Como ya se ha indicado, una Red Bayesiana es un grafo acíclico dirigido. Se puede interpretar, por tanto, una Red Bayesiana de dos formas:

- Distribución de probabilidad: Representa la distribución de la probabilidad conjunta de las variables representadas en la red.

- Base de reglas: cada arco representa un conjunto de reglas que asocian a las variables 
- involucradas. Dichas reglas están cuantificadas por las probabilidades respectivas.

La estructura de una Red Bayesiana se puede determinar de la siguiente manera:

1) Se asigna un vértice o nodo a cada variable (Xj) y se indica de qué otros vértices es una causa directa; a ese conjunto de vértices "causa del nodo (Xj)" se lo denota como el conjunto $\pi \mathrm{Xj}$ y se lo llamará "padres de Xj". 2) Se une cada padre con sus hijos con flechas que parten de los padres y llegan a los hijos.

3) A cada variable $X j$ se le asigna una matriz $P(X j \mid \pi X j)$ que estima la probabilidad condicional de un evento $\mathrm{Xj}$ $=x \mathrm{j}$ dada una combinación de valores de $\operatorname{los} \pi \mathrm{Xj}$. Las Redes Bayesianas permiten definir modelos y utilizarlos tanto para hacer razonamiento de diagnóstico (pues obtienen las causas más probables dado un conjunto de síntomas), como para hacer razonamiento predictivo (obteniendo la probabilidad de presentar un cierto síntoma suponiendo que existe una causa conocida). Una de las características de las Redes Bayesianas es que un mismo nodo puede ser fuente de información u objeto de predicción dependiendo de cuál sea la evidencia disponible. A continuación se muestran cuáles son las características de estos dos tipos de inferencia utilizando una Red Bayesiana.

\section{Discretización de variables}

Si los atributos no siguen una distribución gaussiana, la alternativa es convertirlos a discretos agrupando los valores en un conjunto de rangos o intervalos. No obstante, a menudo conviene representar un fenómeno continuo en la naturaleza usando variables discretas. Para ello, las medidas continuas tienen que ser discretizadas. Esto puede hacerse proyectando la escala de valores continua en un conjunto finito de intervalos. Los valores que caigan en el mismo rango se considerarán como un mismo estado.

La discretización consiste en dividir el rango de las variables continuas en un número finito de intervalos exhaustivos y exclusivos. Por ejemplo, si la variable calado de un muelle en un puerto a modelar puede tomar cualquier valor entre 6 y 20 , es posible dividir el rango en un número finito de intervalos: (6-12], (12-14] y (14-20). Naturalmente, al discretizar se pierde información que depende del dominio y el número de intervalos. Es el método más común ya que la mayoría de las herramientas y algoritmos se basan en nodos discretos.
Para el proceso de construcción de los modelos, una vez seleccionadas las variables de estudio en las tareas anteriores, es necesario proceder a la discretización de las variables. Normalmente las redes bayesianas consideran variables discretas o nominales, por lo que si no lo son, hay que discretizarlas antes de construir el modelo, pues, aunque existen modelos de redes bayesianas con variables continuas, éstos están limitados a variables gaussianas y relaciones lineales.

Existen dos tipos de técnicas de discretización:

- No supervisada: no considera la clase, distinguiéndose entre:

- Intervalos iguales

- Intervalos con los mismos datos

- En base al histograma

- Supervisada: en base a la clase. Considerando los posibles "cortes" entre clases. Este tipo de discretización supone un problema de complejidad computacional.

- Probar clasificador (con datos diferentes)

- Utilizar medidas de información (p. ej., reducir la entropía)

\section{Construcción de los modelos}

Después de definir las variables, el siguiente paso en la construcción de un modelo es definir su estructura. Esto e hace conectando variables con arcos (también llamados enlaces). En las redes bayesianas los arcos son dirigidos. Cambiar la dirección de un arco cambia su significado. La ausencia de un arco entre dos variables indica que no existen relaciones de dependencia directa entre ellas, sino a lo sumo a través de otras variables. La presencia de un arco indica una relación de influencia causal entre dos variables.

En esta parte del proyecto, el aprendizaje estructural consiste en encontrar las relaciones de dependencia entre las variables, de forma Que se pueda determinar la topología o estructura de la red bayesiana. De acuerdo al tipo de estructura, se aplican diferentes métodos de aprendizaje estructural: aprendizaje de árboles, aprendizaje de poli-árboles, aprendizaje de redes multiconectadas, métodos basados en medidas y búsqueda, métodos basados en relaciones de dependencia.

Para la construcción del modelo, se ha empleado el algoritmo K2, el cual está basado en la optimización de una medida, que es lo que se pretende en la planificación, optimizar los ratios de explotación. Esa medida se usa 


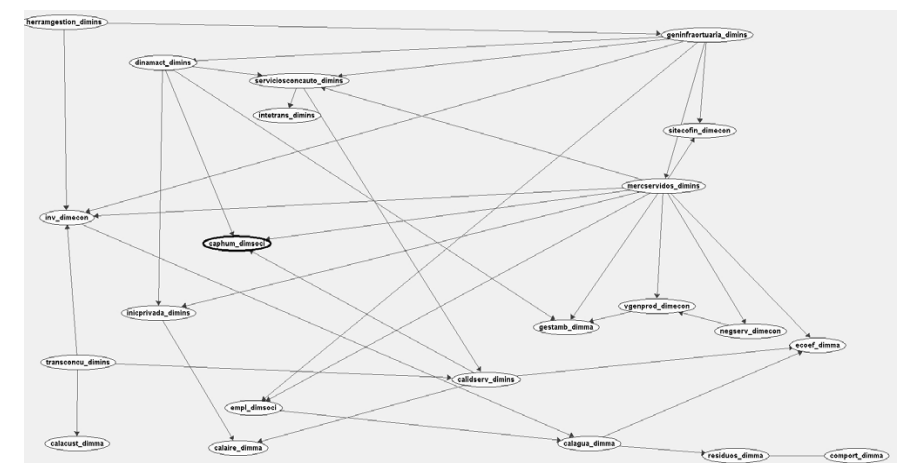

Figura 4. Red Bayesiana. Algoritmo K2.

para explorar, mediante un algoritmo de ascensión de colines, el espacio de búsqueda formado por todas las redes que contienen las variables de la base de datos. Se parte de un red inicial y ésta se va modificando (añadiendo arcos, borrándolos o cambiándolos de dirección), obteniendo una nueva red con mejor medida. En concreto, la medida K2 [38] para una red $\mathrm{G}$ y una base de datos $\mathrm{D}$ es la que se obtiene a partir de la Ecuación siguiente (1):

$$
f(G: D)=\log P(G)+\sum_{i=1}^{n}\left[\sum_{k=1}^{s i}\left[\frac{\log \left(\Gamma\left(\eta_{i k}\right)\right)}{\Gamma\left(\eta N_{I K}+\eta_{I K}\right)}+\sum_{j=1}^{r i} \frac{\log \left(\Gamma\left(\eta N_{i j k}+\eta_{i j k}\right)\right)}{\Gamma\left(\eta_{i j k}\right)}\right]\right]
$$

donde $\mathrm{N}_{\mathrm{ikj}}$ es la frecuencia de las configuraciones encontradas en la base de datos D de las variables xi, donde $\mathrm{n}$ es el número de variables, tomando su j-ésimo valor y sus padres en $\mathrm{G}$ tomando su k-ésima configuración, donde si es el número de configuraciones posibles del conjunto de padres y $r_{i}$ es el número de valores que puede tomar la variable $\mathrm{x}_{\mathrm{i}}$.

\section{Clasificación}

El último paso en el proceso de modelado es especificar parámetros, basta con proporcionar las probabilidades a priori de los nodos raíz y las probabilidades condicionales del resto de los nodos.

En esta fase se aprovechan las características que poseen los métodos bayesianos en tareas de aprendizaje. Cada ejemplo observado va a modificar la probabilidad de que la hipótesis formulada sea correcta (aumentándola o disminuyéndola). Es decir, una hipótesis que no concuerda con un conjunto de ejemplos significativos no es desechada por completo sino que se disminuirá la probabilidad estimada para la hipótesis. Los métodos bayesianos permitirán tener en cuenta en la predicción de la hipótesis el conocimiento a priori o conocimiento del dominio en forma de probabilidades.

\section{RESULTADOS}

Es necesario especificar dos aspectos para describir una Red Bayesiana: topología de grafo (estructura) y los parámetros de cada Distribución Condicional de Probabilidad (CPD). Esto hace posible generar ambos a partir de los datos. Sin embargo, definir la estructura es más difícil que definir los parámetros. Además, cuando algunos de los nodos están ocultos o faltan datos, definirlo es más difícil que definirlo cuando se observa todo.

LaRed Bayesianaes desconocidaen muchos de los sucesos prácticos y, por tanto, se necesita definir a partir de los datos. Este problema se conoce como problema de definición de una Red Bayesiana. Describiéndolo de una forma informal, sería: obtención de los datos base y priorización de la información (por ejemplo, conocimiento del experto, relación causal); estimación de la topología del grafo (estructura de la red) y distribución de los parámetros JPD (parámetros de probabilidad conjunta) en la Red Bayesiana.

El desarrollo de la estructura de una Red Bayesiana se considera un problema más complejo que la definición de los parámetros de dichared. Asimismo, existe otro handicap en el caso de situaciones de observación parcial cuando los nodos están ocultos o cuando directamente faltan.

El caso simple es una Red Bayesiana definida y detallada por un experto. Por lo que se usa para desarrollar inferencias. En otras aplicaciones, la tarea de definir la red es demasiado compleja, siendo necesario que la estructura de la red y la distribución local de los parámetros sea desarrollada a partir de datos.

Automáticamente, el desarrollo de la estructura gráfica de una Red Bayesiana es un reto que se persigue dentro del aprendizaje de la máquina.

La red construida es la que se muestra en la Figura 4. Red obtenida a partir del algoritmo K2. 
Como se aprecia ninguna variable ha quedado descolgada: Los pasos que son necesarios seguir son los siguientes:

- Identificación de los factores relevantes.

- Determinación de cómo esos factores están relacionados causalmente entre ellos. El arco causa-efecto significa que la causa es un factor involucrado en provocar ese efecto

A modo de ejemplo, las relaciones más significativas se muestran en el caso siguiente. En él, en el caso de las variables padres, inv_dimecon y geoinfraortuaria dimins, es el nodo de herramgestion_dimins. Cuando se da herramgestion_dimins, entonces inv_dimecon y geoinfraortuaria_dimins, son condicionalmente independientes (Figura 5).

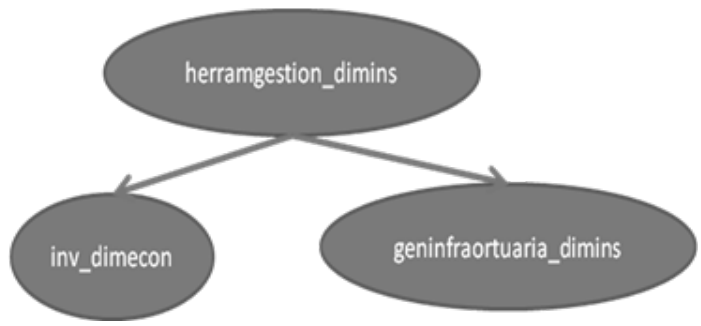

Figura 5. Relación 1.

La variable herramgestion_dimins es una variable decisora, aparece en la red como una variable "nodo" de la que salen arcos, por lo que se genera una conexión divergente, se tiene un nodo padre que proyecta todos sus arcos hacia varios hijos. O lo que es lo mismo las flechas salen de él y divergen hacia sus hijos. (Figura 5).

Cuando se conoce el estado de la variable padre existe una dependencia entre las variables, sin embargo, cuando el estado de la variable padre no se conoce, las variables hijo se tornan independientes y la información no se propaga por la red si se añaden evidencias sobre los nodos hijos. (Figura 5).

Un efecto que tiene dos o más arcos de entrada desde otros vértices se considera como un efecto común de esas causas. Una causa que tiene dos o más arcos de salida a otros vértices se considera como una causa común (factor) de esos efectos. Los efectos de una causa común son, normalmente, observables.

Siguiendo la suposición de independencia de la Red Bayesiana, en este caso, se observan varias declaraciones de independencia respecto de cada uno de los factores.
Cuando mercservidos_dimins se introduce, sitecofin dimecon, serviciosconcauto_dimins, inicprivada_dimins, ecoef_dimma, vgenprod_dimecon, empl-dimsoci, inv_dimecon, negserv_dimecon, gestamb_dimma, and caphum_dimensoci son independientes condicionalmente o su antecesor geoinfraortuaria_dimins. (Figura 6).

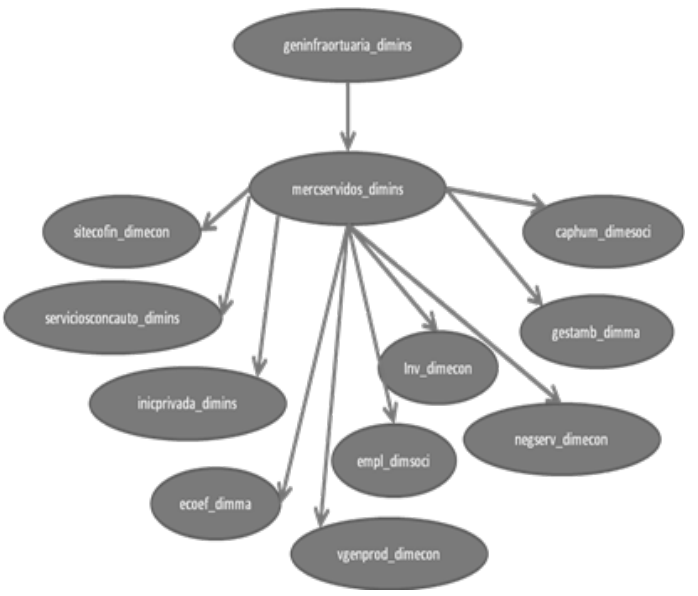

Figura 6. Relación 3.

Grafo casual: la variable mercservidos_dimins tiene diez efectos comunes: sitecofin_dimecon, serviciosconcauto_dimins, inicprivada_dimins, ecoef_dimma, vgenprod_dimecon, empl-dimsoci, inv_dimecon, negserv_dimecon, gestamb_dimma, and caphum_dimensoci (Figura 6).

\section{CONCLUSIONES}

De la red obtenida mediante al empleo del algoritmo K2 se aprecia que la categoría más decisora en la sostenibilidad es la categoría institucional, siendo las variables de las dimensiones económica, social y ambiental son efecto de variables de esta dimensión. Ordenando el resto de categorías por capacidad decisora, se observa que a la dimensión institucional le siguen la económica y social a un mismo nivel, quedando en último lugar la dimensión ambiental.

De igual forma, las variables de la categoría dimensión institucional se encuentran muy relacionadas entre sí. Dentro de las variables institucionales, los sistemas de gestión de apoyo a la toma de decisiones, formados por los sistemas de gestión de la calidad, cuadros de mando integral, campañas de caracterización de mercados, etc., quedan representados en la red por la variable herramgestion_dimins, la cual se presenta como una variable padre de la red. 
Lo mismo ocurre con la variable transconcu_dimins que representa a las iniciativas dirigidas a garantizar que todo operador que desee prestar servicios en el puerto u optar a una concesión pueda conocer de modo transparente las condiciones para operar en el puerto y los mecanismos administrativos que regulan dicho proceso. Dicha variable es también una variable decisora de la red.

Otra variable fundamental en la estructura de la red es mercservidos_dimins, pues de ella salen 10 fechas a 10 nodos diferentes (Tabla 3), siendo estos los efectos de la estructura y evolución de los principales tráficos de mercancías, considerando como tales a los efectos tanto sociales, económicos, institucionales y medioambientales. Así, se puede decir que los mercados servidos tienes efectos, dentro de la categoría institucional, sobre el marco de prestación y regulación de los servicios del puerto, así como sobre el número de empresas que operan en el puerto. De igual forma, en la categoría económica, dicha variable tiene efectos sobre el EBIDTA, EBIDTA/tonelada, sobre inversión pública en relación con el cash-flow y sobre los ingresos por tasas de ocupación y actividad entre otros; y en la categoría social ejerce un efecto sobre sus dos variables, las cuales representan el empleo en la comunidad portuaria, la seguridad laboral y formación en servicios y concesiones portuarios, empleo, comunicación interna y la participación, formación, estructura de plantilla y equidad, seguridad y salud en el trabajo. En el caso de la categoría medioambiental, los mercados servidos son la causa de los grados de implantación de los sistemas de gestión ambiental (EMAS, ISO 14001 y PERLS) y recursos económicos invertidos gastos, así como de inversiones, asociados a la implantación, certificación y mantenimiento de un sistema de gestión ambiental y de la eficiencia en el uso del suelo, consumo de agua y energía eléctrica por la Autoridad Portuaria.

Tabla 3. Hijos de la variable mercados servidos perteneciente a la dimensión institucional (mercservidos_dimins)

\begin{tabular}{|c|c|}
\hline Hijo de mercservidos_dimins & Descripción \\
\hline serviciosconcauto_dimins & $\begin{array}{c}\text { Tipos, marco de prestación y } \\
\text { regulación }\end{array}$ \\
\hline inicprivada_dimins & $\begin{array}{c}\text { Número de empresas que operan } \\
\text { en el puerto, superficie terrestre } \\
\text { ocupada, caracterizada como uso } \\
\text { comercial concesionado, etc. }\end{array}$ \\
\hline vgenprod_dimecon & $\begin{array}{c}\text { Entre otros indicadores: } \\
\text { productividad del trabajo según } \\
\text { ingresos, productividad del } \\
\text { trabajo según EBIDTA, etc. }\end{array}$ \\
\hline
\end{tabular}

\begin{tabular}{|c|c|}
\hline Hijo de mercservidos_dimins & Descripción \\
\hline sitecofin_dimecon & $\begin{array}{c}\text { Entre otros indicadores: } \\
\text { rentabilidad sobre activos, } \\
\text { EBIDTA/tonelada, servicio } \\
\text { de la deuda, relación gastos } \\
\text { de explotación e ingresos de } \\
\text { explotación, etc. }\end{array}$ \\
\hline inv_dimecon & $\begin{array}{c}\text { Entre otros indicadores: } \\
\text { inversión pública en relación } \\
\text { con el cash-flow, inversión ajena } \\
\text { frente a la inversión pública, } \\
\text { renovación de activos }\end{array}$ \\
\hline negserv_dimecon & $\begin{array}{c}\text { Entre otros indicadores: ingresos } \\
\text { por tasas de ocupación y } \\
\text { actividad, uso comercial de la } \\
\text { superficie, uso de los muelles, etc. }\end{array}$ \\
\hline empl_dimsoci & $\begin{array}{c}\text { Empleo en la comunidad } \\
\text { portuaria, seguridad laboral } \\
\text { y formación en servicios y } \\
\text { concesiones portuarios, etc. }\end{array}$ \\
\hline caphum_dimensoci & $\begin{array}{l}\text { Empleo, comunicación interna y } \\
\text { participación, formación, estructura } \\
\text { de plantilla y equidad, seguridad y } \\
\text { salud en el trabajo, etc. }\end{array}$ \\
\hline gestamb_dimma & $\begin{array}{l}\text { Grados de implantación de los } \\
\text { sistemas de gestión ambiental } \\
\text { (EMAS, ISO 14001 y PERLS) y } \\
\text { recursos económicos invertidos } \\
\text { gastos, así como inversiones } \\
\text { en su caso, asociados a la } \\
\text { implantación, certificación y } \\
\text { mantenimiento de un sistema de } \\
\text { gestión ambiental }\end{array}$ \\
\hline ecoef_dimma & $\begin{array}{c}\text { Eficiencia en el uso del suelo, } \\
\text { consumo de agua y energía } \\
\text { eléctrica por la Autoridad Portuaria }\end{array}$ \\
\hline
\end{tabular}

Por lo tanto, los mercados servidos suponen una variable muy importante dentro de la planificación desde una perspectiva sostenible al ejercer influencia en variables de todas las categorías.

Por otro lado, las variables económicas resultan fundamentales en términos de causa-efecto, pues se trata de variables que son efecto de la variable mercados servidos que pertenece a la dimensión institucional. De ello se desprende que el valor generado y la productividad son dependientes del negocio y servicios (ingresos por tasas de ocupación y actividad, uso comercial de la superficie, uso de los muelles, etc.).

De igual forma, las variables de la dimensión social son efecto de variables de la dimensión institucional, pero, sin embargo, no tienen relación directa entre ellas, es decir, no encuentra una relación directa entre las dos variables de la dimensión social: capital humano de la actividad portuaria y empleo y seguridad laboral en la comunidad portuaria.

Finalmente, las variables de la categoría ambiental se encuentran muy relacionadas entre sí en la red 
bayesiana definida, siendo efectos de variables de la categoría institucional, principalmente. Por ello, aunque las Autoridades Portuarias no tienen competencias ambientales, desempeñan un papel clave en la adecuada gestión ambiental del puerto al actuar como gestores de infraestructura, reguladores, coordinadores de los servicios prestados y, en especial, como líderes de la comunidad. La actividad portuaria genera impactos tanto en el medio acuático como en el terrestre y el aéreo, siendo este capítulo el que evalúa los impactos y las medidas implantadas para reducirlos. Asimismo, también da información sobre los sistemas de recepción y gestión de quejas ambientales que se encuentran disponibles en todos los puertos del Sistema Portuario Español.

En vista de los resultados se concluye que un aspecto clave en la planificación y gestión portuaria es que las Autoridades Portuarias vayan incorporando los elementos exigibles en materia de sostenibilidad que la ley de Puertos contempla a los instrumentos de los que disponen para la regulación de sus servicios y la gestión del dominio público.

Es por ello que el Organismo Público Puertos del Estado no realiza la planificación y gestión portuaria guiándose únicamente por la viabilidad económica de su actividad y la contribución a la organización de una logística eficiente, sino que también integra el resto de dimensiones de la sostenibilidad, tal y como queda patente en la responsabilidad que muestra con la sociedad y el medio ambiente, así como en su compromiso con la transparencia; constituyendo las memorias de sostenibilidad un claro testimonio de ello.

\section{REFERENCIAS}

[1] O. Doerr. "Políticas portuarias sostenibles". Boletín FAL. CEPAL. Edición nº 299, número 7 de 2011

[2] Global Reporting Initiative. "Guía para la elaboración de Memorias de Sostenibilidad". Versión 3.1. GRI. 2000

[3] O. Serrano. "Operativa portuaria y sostenibilidad". CONAMA LOCAL 2015, 7 octubre de 2015. Málaga

[4] M.Feito Cespon, R. Cespon CastroandM.A.Rubio Rodriguez. "Optimization model to sustainable design of multiple-products recycling supply chain". Ingeniare. Rev. chil. ing. [online]. Vol. 24
$\mathrm{N}^{\circ} 1$, pp.135-148. 2016. ISSN 0718-3305.http:// dx.doi.org/10.4067/S0718-33052016000100013.

[5] R. J. Sánchez, A. Jauimurzono, G. Willmsmeier, G. Pérez Salas, O. Doerr y F. Pinto. "Transporte marítimo y Puertos. Desafío y oportunidades en busca de un desarrollo sostenible de América Latina y El Caribe". CEPAL. Serie Recursos Naturales e Infraestructura. 2015

[6] C. Crespo Soler, A. Giner Fillol, J.A. Morales Baraza, N. Ponte Tubal y V.M. Ripoll Feliu. "La información de sostenibilidad en el marco de las cuentas anuales: análisis aplicado al caso de la Autoridad Portuaria de Valencia". Revista do Contabilidado de Maestrado em Ciências Contábeis da UERJ, Rio Janeiro, Vol.12 ํ3, pp.11. 2007, p-11 set./dez, 2007

[7] Grupo De Trabajo 23. "La sostenibilidad en los puertos". CONAMA VII Cumbre del desarrollo sostenible, 24 de noviembre de 2004

[8] C. Crespo Soler, V.M. Ripoll Feliu,A.M. Crespo Trujillo y A. Giner Fillol. "La sostenibilidad ambiental en el sistema portuario de titularidad estatal". XIII Congreso AECA. Armonización y Gobierno de la Diversidad. 22-24 Septiembre 2005.

[9] F. González, A. Guerra, F. Martín, J. J. Nóvoa, C. Otero y J.Penela. "Medición de la sostenibilidad en el sistema portuario Español: propuesta metodológica a través de indicadores sintéticos de desarrollo sostenible". XII Reunión de economía mundial, mayo de 2010. Santiago de Compostela.

[10] Autoridad Portuaria De A Coruña, Autoridad Portuaria De Valencia, Organismo Público Puertos Del Estado. "Guía para la elaboración de memorias de sostenibilidad en el sistema portuario español". FEPORTS. 2008

[11] Puertos Del Estado. "Memoria de sostenibilidad del sistema portuario de interés general". 2011

[12] N. Friedman and M. Goldszmidt. "Building classifiers using bayesian networks. Proceedings of the National Conference on Artificial Intelligence", Menlo Park, Ca: AAAI Press. 1996

[13] S. Jara-Díaz, E. Martínez-Budría, C. Cortes and A. Vargas. "Marginal costs and scale economies in Spanish ports". 25th European Transport Forum, Proceedings Seminar L, PTRC, London, pp. 137-147. 1997

[14] C. Tebaldi and M. West. "Bayesian inference on network traffic using link count data". Journal of the American Statistical Association, Vol. 93 (442), pp. 557-573. 1998 
[15] J. Cain. "Planning improvements in natural resource management. guidelines for using bayesian networks to support the planning and management of development programmes in the water sector and beyond". Wallingford, Oxon: CEH Wallingford. 2001

[16] C. Conati, A. Gertner and K. Vanlehn. "Using bayesian networks to manage uncertainty in student modeling". User Modeling and UserAdapted Interaction, Vol.12, pp. 371-417. 2002

[17] J. Bromley, N. A. Jackson, O. J. Clymer, A. M. Giacomello and F. V. Jensen. "The use of hugin ${ }^{\circledR}$ to develop bayesian networks as an aid to integrated water resource planning" 1. Environmental Modelling \& Software, Vol.20 (2), pp. 231-242. 2005

[18] S. Sun, C. Zhang and G. Yu. "A bayesian network approach to traffic flow forecasting". Intelligent Transportation Systems, IEEE Transactions on, 7(1), 124-132. 2006

[19] W. Zheng, D. H. Lee and Q. Shi. "Short-term freeway traffic flow prediction: Bayesian combined neural network approach". Journal of transportation engineering, Vol.132 (2), pp.114-121. 2006

[20] D. Janssens, G. Wets, T. Brijs, K. Vanhoof, T. Arentze and H. Timmermans, H. "Integrating Bayesian networks and decision trees in a sequential rule-based transportation model". European Journal of operational research, Vol. 175 (1), pp. 16-34. 2006

[21] E. Castillo, J. M. Menéndez and S. SánchezCambronero. "Traffic estimation and optimal counting location without path enumeration using Bayesian networks". Computer-Aided Civil and Infrastructure Engineering, 23(3), 189-207. 2008

[22] E. Castillo, J. M. Menéndez \& S. SánchezCambronero. "Predicting traffic flow using Bayesian networks". Transportation Research Part B: Methodological, Vol. 42 No. 5; 482-509. 2008

[23] P. Trucco, E. Cagno, F. Ruggeri and O. Grande. "A Bayesian Belief Network modelling of organisational factors in risk analysis: A case study in maritime transportation". Reliability Engineering \& System Safety, 93(6), 845-856. 2008

[24] E. Klemola, J. Kuronen, J. Kalli, T. Arola, T., M. Hanninen, A. Lehikoinen and U. Tapaninen. "A cross-disciplinary approach to minimising the risks of maritime transport in the Gulf of Finland". World Review of Intermodal Transportation Research, Vol. 2, No. 4, 343-363. 2009

[25] P. Kaluza, A. Kölzsch, M. T. Gastner \& B. Blasius. "The complex network of global cargo ship movements". Journal of the Royal Society Interface, rsif20090495. 2010

[26] A. Hofleitner, R. Herring, P.Abbeel and A. Bayen. "Learning the dynamics of arterial traffic from probe data using a dynamic Bayesian network". Intelligent Transportation Systems, IEEE Transactions on, 13(4), 1679-1693. 2012

[27] N. G. Cancelas, F. S. Flores and A. C. Orive. "Modelo de eficiencia de las terminales de contenedores del sistema portuario español". Revista Electrónica de Comunicaciones y Trabajos de ASEPUMA. Rect@. Vol. 14; 49-67. 2013

[28] A. Camarero, N. González-Cancelas, F. Soler and I. López. "Utilización de redes bayesianas como método de caracterización de parámetros físicos de las terminales de contenedores del sistema portuario español". Revista de Ingeniería. Vol. 39, pp. 31-38. 2013

[29] F. S. Flores, N. G. Cancelas, A. C. Orive, J. L. A. Gárate and M. D. C. P. Monzón. "Diseño de un modelo de planificación de zonas de actividades logísticas mediante el empleo de redes bayesianas". Revista Ingeniería Industrial, 12(1). 2014

[30] K. X.Li, J. Yin, H.S. Bang, Z. Yang and J. Wang. "Bayesian network with quantitative input for maritime risk analysis". Transportmetrica A: Transport Science, 10(2), 89-118. 2014

[31] T. Rodríguez García, N. González Cancelas, and Soler-Flores, F. "Setting the Port Planning Parameters In Container Terminals through Bayesian Networks". PROMET-Traffic\&Transportation, 2015, vol. 27, no 5, p. 395-403.

[32] Rodríguez García, T. "Aplicaciones tecnológicas en la logística de transportes portuarios. Las terminales de contenedores". Revista Transporte y Territorio, 2016, no 14.

[33] J. Pearl. "Probabilistic reasoning in intelligent systems: Networks of plausible inference". Morgan Kaufmann. 1998

[34] J.L.Almazán-Gárate, M. C.Palomino-Monzón, N. González-Cancelas and F. Soler-Flores. "Relationship between air pollution and natural gas with respect to maritime transport. 
Methodology based on Bayesian Networks". Global Virtual Conference. 7-11 April 2014. Transport and Logistics Section.

[35] E. Castillo, J. M. Gutiérrez and A. S. Hadi. "Expert Systems and Probabilistic Network Models". Springer Verlag, New York. 1997

[36] R. O. Duda, P. E. Hart and D. G. Stork. "Pattern Classification". Wiley, New York. 2001
[37] J. Pearl. "The Solution for the Branching Factor of the Alpha-Beta Pruning Algorithm and its Optimality". Communications of the ACM. 1982. Vol 25, no.8

[38] G. F. Cooper and E. Herskovitz. "A bayesian method for the induction of probabilistic networks from data". Machine Learning, Vol.9 (4), pp. 309-348.1992 